\title{
STOCHASTIC CONTROL FOR LINEAR DISCRETE-TIME DISTRIBUTED-LAG MODELS
}

\author{
W. Brian Arthur
}

RR-77-18

August 1977

Research Reports provide the formal record of research conducted by the International Institute for Applied Systems Analysis. They are carefully reviewed before publication and represent, in the Institute's best judgment, competent scientific work. Views or opinions expressed therein, however, do not necessarily reflect those of the National Member Organizations supporting the Institute or of the Institute itself.

In ternational Institute for Applied Systems Analysis

A-2361 Laxenburg, Austria 



\section{PREFACE}

Models with distributed-delay variables arise in many subjects of interest to IIASA. They occur for example in economic planning as the distributed-lag policy model, in time-series analysis as the ARIMA process, and in population and agricultural planning as the age-dependent regenerative process. Derivation of optimal estimation and control procedures for such models is the subject of this paper. 



\section{Stochastic Control for Linear \\ Discrete-Time Distributed-Lag Models}

\section{INTRODUCTION}

An important class of linear-quadratic Gaussian problems has lagged variables in the dynamics or the observations: problems where process behavior depends on the past trajectory for example, where control action is retarded, or where information is delayed. For such problems in continuous time a fairly comprehensive theory is available (see for example Koivo (1974), Kwong and Willsky (1977), Arthur (1977)); for discrete time no satisfactory comprehensive theory as yet exists, but certain procedures are available for numerical solution (Chow (1975), Aoki (1976)).

Both the Chow and the Aoki procedures redefine the state vector to one of higher dimension to transform the original lagged problem into an equivalent, but larger, non-lagged problem. Standard results then apply. While these methods are convenient they suffer drawbacks. Transition matrices for the equivalent problem are large and sparse, with side dimension $N$ determined by the duration of the longest lags. Calculation of the Riccati sequence then requires operations of order $N^{3}$ at each step. Also, since results are expressed in terms of the new, non-lagged problem, much of the special structure of the time-lag controller and estimator is obscured.

It would be better from both computational and theoretical points of view to derive results in terms of the original problem and in non-sparse form. For continuous-time problems this is possible, using the so-called Carathéodory and maximum-principleFredholm techniques. These, however, are ill-suited to discrete time and to problems with delays in the control: we cannot apply them here. One way to derive non-sparse results for discrete-time 
delay problems would be to use a direct dynamic programming argument (see Arthur (1977)). A second and yet more straightforward derivation is proposed in this paper. We translate the problem into equivalent non-lagged form and apply standard theory, then use careful matrix partitioning to reexpress the solution in terms of the variables and matrices of the original problem. The results are then in the non-sparse form we want: the qualitative structure of the time-lag controller and estimator stands out clearly; Riccati calculations are reduced to order $N^{2}$; and the discretetime Riccati equations correspond almost term for term to those for the known continuous-time case--the connection between the two becomes clear.

The problem treated is general: distributed lags may occur in dynamics and observations in both state and control variables. Results apply not only to design of discrete-time filters and controllers, but to numerical solution of continuous-time problems which are discretized at the outset.

\section{THE DISTRIBUTED-LAG PROBLEM}

We study linear processes that evolve according to the distributed-lag dynamics:

$$
x_{i+1}=\sum_{\theta=0}^{k} A_{i}(\theta) x_{i-\theta}+\sum_{\theta=1}^{h} B_{i}(\theta) u_{i-\theta}+C_{i} u_{i}+\omega_{i},
$$

where a linear measurement of past states and controls is available:

$$
z_{i}=\sum_{\theta=0}^{k} H_{i}(\theta) x_{i-\theta}+\sum_{\theta=1}^{h} G_{i}(\theta) u_{i-\theta}+\psi_{i} \text {. }
$$

The distributed-lag dynamics of this process include single lags as a special case, and the observations include pure informational delay as a special case. The usual notation applies: $x$ is an $\mathrm{n}$-dimensional vector describing the state, $\mathrm{u}$ an m-dimensional vector of policy instruments, $z$ a p-dimensional vector of observations. The parameter matrices are assumed known and nonrandom. All disturbance or error vectors throughout the paper, unless 
stated otherwise, are distributed normally, are independent of each other, and have zero mean. Expectations E[ ] are taken over appropriate states, observations, and, where necessary, controls. $I_{n}$ will denote an identity matrix of dimension $n$. The process disturbance $\omega_{i}$ and measurement error $\psi_{i}$ have variances $\Omega_{i}$ and $\psi_{i}$ (the latter matrix is assumed positive definite). Initial values $\mathrm{x}_{0}, \ldots, \mathrm{x}_{-\mathrm{k}}$, and $\mathrm{u}_{-}, \ldots, \mathrm{u}_{-\mathrm{h}}$, are assumed to be distributed normally with given means and variances. Subsequent estimation is conditioned on this initial information.

We wish to choose controls $u_{i}$ at times 0 to $T-1$ to minimize

$$
J=E\left[\sum_{i=0}^{\top 1}\left(x_{i}^{\prime} Q_{0} x_{i} x_{i}+u_{i}^{\prime} R_{i} u_{i}\right)+x_{T}^{\prime} Q_{0}{ }_{T} x_{T}\right],
$$

where the expectation $\mathrm{E}$ is taken over all states and observations; $Q_{0}$ is assumed positive semidefinite and $R$ positive definite. $z_{i}$ will denote $\left\{z_{0}, \ldots, z_{i}\right\}$, the information available at time $i$.

In most applications the implementation of controls is imperfect. The actual value of the controls $u_{i}$ will deviate from the intended value $u_{i}$ as in

$$
u_{i}=u_{i}+v_{i},
$$

where implementation error $v_{i}$ has variance $\tau_{i}$. Usually there is no need to consider this type of error separately--it can be subsumed into general process error by substituting the intended for the actual policy value in the dynamics. With lags in the control, however, this procedure would cause sequential correlation of process errors. Instead we substitute the intended control only partially into the dynamics, by writing

$$
\mathbf{x}_{i+1}=\sum_{\theta=0}^{k} A_{i}(\theta) x_{i-\theta}+\sum_{\theta=1}^{h} B_{i}(\theta) u_{i-\theta}+C_{i} u_{i}+\lambda_{i}
$$

with

$$
\lambda_{i}=\omega_{i}+c_{i} v_{i}
$$


Composite error, $\lambda_{i}$, now has mean zero and variance $\Omega_{i}+C_{i}{ }^{T} C_{i}^{\prime}$. We thus retain the advantages of sequentially uncorrelated process noise, $\lambda_{i}$, and perfectly known control, $u_{i}$, at the price of including past controls which are not perfectly known. These must be estimated, as must the state, at each step. (Note that the problem is unchanged by substituting $u_{i}$ for $u_{i}$ in the performance criterion. $\quad E\left[\left[u_{i}^{\prime} R_{i} u_{i}\right]+\sum \operatorname{Tr}\left(R_{i} T_{i}\right)\right.$ replaces $E\left[\left[u_{i}^{\prime} R_{i} u_{i}\right]\right.$ and since the trace term is constant it does not affect the solution.)

\section{THE EQUIVALENT PROBLEM AND SOLUTION}

To solve the problem, we first translate it to an equivalent non-lagged form and apply standard results. Define $y_{i}$, the history of the system at time $i$, to be

$$
\left[x_{i}^{\prime}, \ldots, x_{i-k}^{\prime} \mid u_{i-1}^{\prime}, \ldots, u_{i-h}^{\prime}\right]^{\prime},
$$

the vector obtained by combining the state history (state lagged variables) with the control history (control lagged variables). We take the history as the new "state" of the equivalent system.

The history evolves according to

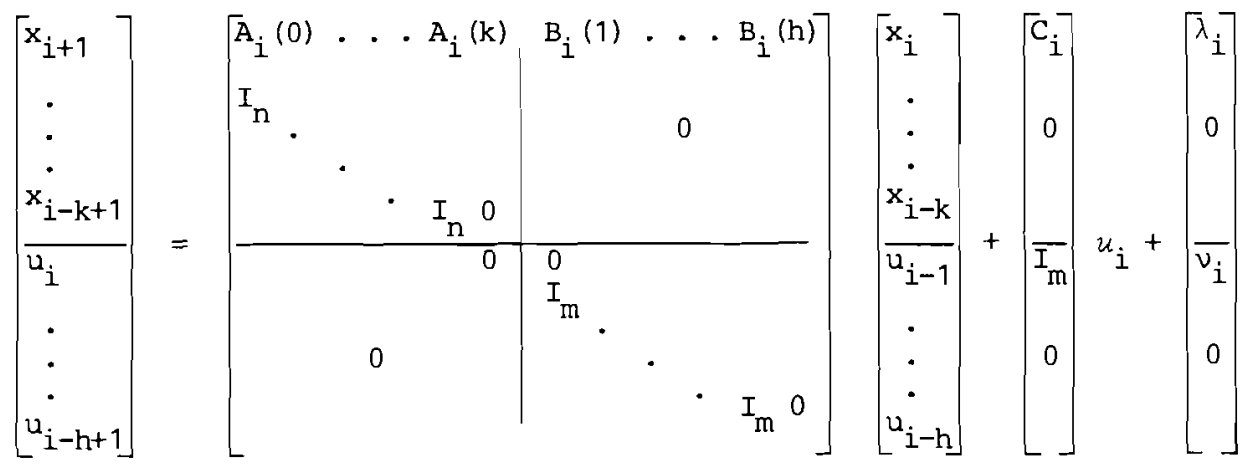

with observations 


$$
z_{i}=\left[H_{i}(0), \ldots, H_{i}(k) \mid G_{i}(1), \ldots, G_{i}(h)\right]\left[x_{i}\right]+\psi_{i} .(8)
$$

Writing the history vector as $y_{i}$, the problem is now in the standard non-lagged form

$$
\begin{aligned}
y_{i+1} & =\tilde{\Phi}_{i} y_{i}+\tilde{C}_{i} u_{i}+\xi_{i}, \\
z_{i} & =\tilde{H}_{i} Y_{i}+\psi_{i},
\end{aligned}
$$

where $\xi_{i}$ has variance $\Xi_{i}$.

It remains to rewrite the criterion in this form. Define $Q_{i}$ (positive semidefinite) to be

$\left[\begin{array}{c|c|c}Q_{0} & 0 & 0 \\ \hline 0 & 0 & 0 \\ \hline 0 & 0 & 0\end{array}\right]$

where the partitions are taken to correspond to

$$
\left[x_{i}^{\prime}\left|x_{i-1}^{\prime}, \ldots, x_{i-k}^{\prime}\right| u_{i-1}^{\prime}, \ldots, u_{i-h}^{\prime}\right]
$$

The problem then becomes: choose $u_{i}\left(z_{i}\right)$ to minimize 


$$
J=E\left[\sum_{i=0}^{T-1}\left(Y_{i}^{\prime} Q_{i} Y_{i}+u_{i}^{\prime} R_{i} u_{i}\right)+y_{T}^{\prime} Q_{T} Y_{T}\right] \text {. }
$$

Results for this problem are standard. They may be found for example in Meier, Larson and Tether (1971). For our later use we summarize them briefly here:

1. The optimal control policy is linear in the conditional mean of the state, $\hat{y}_{i \mid i}\left(\equiv \underset{Z_{i}}{E}\left[y_{i}\right]\right.$ : the $\left."\right|_{i}$ " notation means conditioned on all information available at time i):

$$
u_{i}=-\mathrm{P}_{i}^{-1} \mathrm{D}_{\mathrm{i}} \hat{\mathrm{Y}}_{\mathrm{i} \mid \mathrm{i}} \text {. }
$$

The control gain matrices are

$$
\begin{aligned}
& P_{i}=\left(\tilde{C}_{i}^{\prime} K_{i+1} \tilde{C}_{i}+R_{i}\right)>0, \\
& D_{i}=\tilde{C}_{i}^{\prime} K_{i+1} \tilde{\Phi}_{i},
\end{aligned}
$$

where $\mathrm{k}_{i}$ is the solution to the Riccati difference system

$$
\begin{array}{r}
k_{i}=Q_{i}+\tilde{\Phi}_{i}^{\prime} K_{i+1} \tilde{\Phi}_{i}-\tilde{\Phi}_{i}^{\prime} K_{i+1} \tilde{C}_{i}\left(\tilde{C}_{i}^{\prime} K_{i+1} \tilde{C}_{i}+R_{i}\right)-1 \tilde{C}_{i}^{\prime} K_{i+1} \tilde{\Phi}_{i} ; \\
K_{T}=Q_{T} .
\end{array}
$$

2. The conditional mean evolves according to the Kalman filter equation

$$
\hat{y}_{i \mid i}=\hat{y}_{i \mid i-1}+F_{i} g_{i} \text {, }
$$

where $g_{i}$ is the measurement residual

$$
g_{i}=z_{i}-\tilde{H}_{i} \hat{y}_{i \mid i-1} \text {. }
$$

The prediction $\hat{y}_{i \mid i-1}$ is extrapolated from $\hat{y}_{i \mid i}$ by

$$
\hat{y}_{i \mid i-1}=\tilde{\Phi}_{i-1} \hat{y}_{i-1 \mid i-1}+\tilde{C}_{i-1} u_{i-1} ; \hat{y}_{0 \mid-1}=E\left[y_{0}\right] .
$$


The prediction-error covariance matrix, $S_{i} \equiv E\left[\left(y_{i}-\hat{y}_{i \mid i-1}\right)\left(y_{i}-\hat{y}_{i \mid i-1}\right)^{\prime}\right]$, propagates according to

$$
S_{i+1}=\tilde{\Phi}_{i} s_{i} \tilde{\Phi}_{i}^{\prime}+\Xi_{i}-\tilde{\Phi}_{i} S_{i} \tilde{H}_{i}^{\prime}\left(\tilde{H}_{i} s_{i} \tilde{H}_{i}^{\prime}+\psi_{i}\right)^{-1} \tilde{H}_{i} S_{i} \tilde{\Phi}_{i}^{\prime} \text {. }
$$

The optimal filter gain, $F_{i^{\prime}}$ is given by

$$
F_{i}=S_{i} \tilde{H}_{i}^{\prime}\left(\tilde{\mathbf{H}}_{i} S_{i} \tilde{H}_{i}^{\prime}+\Psi_{i}\right)^{-1} \text {. }
$$

We now have a solution in terms of variable $y_{i}$ and sparse matrices $\tilde{\Phi}, \tilde{c}$, etc. In principle the problem is "solved". Note however that computation of $K_{i}$ and $s_{i}$ would require sparse-matrix multiplications of the form $\tilde{\Phi}^{\prime} K \Phi$ at each step (order $(n k+m h+n)^{3}$ multiplications). In the next two sections we reduce such operations significantly and reexpress the above results in terms of the original problem variables and matrices.

\section{OPTIMAL CONTROL POLICY}

In terms of the original problem, the conditional mean $\hat{y}_{i \mid i}$ is reexpressed as $\left[\hat{x}_{i \mid i}^{\prime}, \ldots, \hat{x}_{i-k \mid i}^{\prime} \mid \hat{u}_{i-1 \mid i}^{\prime}, \ldots, \hat{u}_{i-h \mid i}^{\prime}\right]^{\prime}$ where the notation $\hat{\mathbf{x}}_{i-\theta \mid i}$ is read as the estimate of $\mathbf{x}_{i-\theta}$ given all information available at time $i$.

We now partition $K_{i}$ and $D_{i}$ :

$$
\mathrm{K}_{i}=\left[\begin{array}{cc}
\mathrm{K}_{0_{i}} & \mathrm{~K}_{1_{i}} \\
\mathrm{~K}_{1_{i}}^{\prime} & \mathrm{K}_{2}
\end{array}\right] ; \quad \mathrm{D}_{i}=\left[\mathrm{v}_{i} \mid \mathrm{w}_{i}\right] \text {. }
$$

(The submatrices $\mathrm{K}_{0_{i}}$ and $\mathrm{v}_{i}$ correspond to the state history, $\mathrm{x}_{\mathrm{i}}, \ldots, \mathrm{x}_{\mathrm{i}-\mathrm{k}} ; \mathrm{k}_{2}$ and $\mathrm{w}_{\mathrm{i}}$ correspond to the control history, $\left.u_{i-1}, \ldots, u_{i-h} \cdot\right)$

We may now obtain the optimal control law in terms of the matrices of the original problem, by substituting for $D_{i}$ and $\hat{y}_{i \mid i}$ 
in (12). This yields:

$$
u_{i}=-P_{i}^{-1}\left\{\sum_{\theta=0}^{k} v_{i}(\theta) \hat{x}_{i-\theta \mid i}+\sum_{\theta=1}^{h} w_{i}(\theta) \hat{u}_{i-\theta \mid i}\right\} .
$$

The optimal policy is a feedback law, linear in the current estimates of the state and control histories.

By substituting the original problem matrices for $\tilde{\Phi}$ and $\tilde{C}$ in (13) and (14) and multiplying out, we obtain the gain matrices $P_{i}, V_{i}$, and $w_{i}$ :

$$
\begin{aligned}
\mathrm{P}_{i} & =C_{i}^{\prime} \mathrm{K}_{0_{i+1}}(0,0) C_{i}+C_{i}^{\prime} K_{1}(0,1)+K_{i+1}^{\prime}(1,0) C_{i}+K_{2}(1,1)+R_{i+1} \\
v_{i}(\theta) & =C_{i}^{\prime} K_{0}(0,0) A_{i+1}(\theta)+C_{i}^{\prime} K_{0}(0, \theta+1)+K_{i+1}^{\prime}(1,0) A_{i}(\theta)+K_{i+1}^{\prime}(1, \theta+1) \\
w_{i}(\theta) & =C_{i}^{\prime} K_{0}(0,0) B_{i+1}(\theta)+C_{i}^{\prime} K_{1}(0, \theta+1)+K_{1+1}^{\prime}(1,0) B_{i}(\theta)+K_{2}(1, \theta+1) .
\end{aligned}
$$

Finally the Riccati difference system (15) is expanded to yield a recursion for the submatrices $\mathrm{K}_{0}, \mathrm{~K}_{1}, \mathrm{~K}_{2}$ :

$$
\begin{aligned}
& K_{0}(\theta, \phi)=A_{i}^{\prime}(\theta) K_{0}(0,0) A_{i}(\phi)+A_{i}^{\prime}(\theta) K_{0}(0, \phi+1)+K_{0}(\theta+1,0) A_{i}(\phi) \\
& +Q_{0} \delta_{0}(\theta, \phi)+K_{0}(\theta+1, \phi+1)-v_{i+1}^{\prime}(\theta) p_{i}^{-1} v_{i}(\phi) \\
& K_{1}(\theta, \phi)=A_{i}^{\prime}(\theta) K_{0}(0,0) B_{i}(\phi)+A_{i}^{\prime}(\theta) K_{1}(0, \phi+1)+K_{0}(\theta+1,0) B_{i}(\phi) \\
& +k_{1}(\theta+1, \phi+1)-v_{i}(\theta) P_{i}^{-1} W_{i}(\phi) \\
& K_{2}(\theta, \phi)=B_{i}^{\prime}(\theta) K_{0}(0,0) B_{i+1}(\phi)+B_{i}^{\prime}(\theta) K_{1}(0, \phi+1)+K_{1+1}^{\prime}(\theta+1,0) B_{i}(\phi) \\
& +K_{2}(\theta+1, \phi+1)-w_{i}^{\prime}(\theta) P_{i}^{-1} W_{i}(\phi)
\end{aligned}
$$

with end conditions $\mathrm{K}_{0_{\mathrm{T}}}(\theta, \phi)=\mathrm{Q}_{0_{\mathrm{T}}} \delta_{0}(\theta, \phi) ; \mathrm{K}_{1_{\mathrm{T}}}=\mathrm{K}_{2 \mathrm{~T}}=0$. 
(In the above results the indices $\theta, \phi$ are taken over 0 to $k$ or 1 to $h$ as appropriate. The symbol $\delta_{0}(\theta, \phi)=1$ if $\theta$ and $\phi$ are zero; $\delta_{0}(\theta, \phi)=0$ otherwise. Where undefined matrices occur, e.g., $\mathrm{K}_{0}(\mathrm{k}+1,0)$, they are taken as zero.)

The control law parameters may be precomputed. Only the estimates of the lagged variables need then be fed back in real time to determine the optimal control.

In the case of state lags only (where $B(\theta) \equiv 0$ ), the results simplify: $W, K_{1}, K_{2}$ disappear. Where there are control lags only $(A(\theta) \equiv 0), V, K_{1}$, and $K_{0}$ except for $K_{0}(0,0)$ disappear.

\section{THE OPTIMAL FILTER-SMOOTHER}

We now translate the filter results of section 3 to a form that fits the original lagged problem.

$$
\begin{aligned}
& \text { Partition } F_{i} \text { and } S_{i} \text { as } \\
& F_{i}=\left[\begin{array}{c}
M_{i} L_{i}^{-1} \\
N_{i} L_{i}^{-1}
\end{array}\right] ; \quad s_{i}=\left[\begin{array}{cc}
s_{O_{i}} & s_{1} \\
s_{1} & s_{2}
\end{array}\right] \text {, }
\end{aligned}
$$

where $L_{i}$ is defined as

$$
L_{i}=\left[\tilde{H}_{i} S_{i} \tilde{H}_{i}^{\prime}+\Psi_{i}\right]>0 \text {. }
$$

(The submatrix dimensions of $\mathrm{M}_{i}$ and $\mathrm{S}_{0_{i}}$ correspond to the state history, those of $\mathrm{N}_{i}$ and $\mathrm{s}_{2_{i}}$ to the control history.)

Now, substituting for $\hat{y}_{i \mid i}$, the Kalman filter of (16) becomes at each stage an estimator for the history:

$$
\begin{aligned}
& \hat{x}_{i-\theta \mid i}=\hat{x}_{i-\theta \mid i-1}+M_{i}(\theta) L_{i}^{-1} g_{i}, i=0, \ldots, k \\
& \hat{u}_{i-\theta \mid i}=\hat{u}_{i-\theta \mid i-1}+N_{i}(\theta) L_{i}^{-1} g_{i}, \quad i=1, \ldots, h .
\end{aligned}
$$


The history estimates are updated at each stage by combining the previous-stage estimate with the new information $\mathrm{g}_{i^{--t h e y}}$ are improved sequentially as new information comes in, where $g_{i}$ (the residual) is obtained from (17) as

$$
g_{i}=z_{i}-\sum_{\theta=0}^{k} H_{i}(\theta) \hat{x}_{i-\theta \mid i-1}+\sum_{\theta=1}^{h} G_{i}(\theta) \hat{a}_{i-\theta \mid i-1} .
$$

The prediction equation (18) reduces to

$$
\begin{aligned}
\hat{x}_{i+1 \mid i} & =\sum_{\theta=0}^{k} A_{i}(\theta) \hat{x}_{i-\theta \mid i}+\sum_{\theta=1}^{h} B_{i}(\theta) \hat{u}_{i-\theta \mid i}+c_{i} \hat{u}_{i \mid i}, \\
\hat{u}_{i \mid i} & =u_{i},
\end{aligned}
$$

with initial conditions $\hat{x}_{-\theta \mid-1}=E\left[x_{-\theta}\right], \quad \hat{u}_{-\theta \mid-1}=E\left[u_{-\theta}\right]$.

The above equations (24) to (26) make up a recursion system for the estimates of the state and control histories. The filter for the equivalent non-lagged problem has now become a filtersmoother (an estimator of present and past values) for the original lagged problem.

It remains to specify the filter-smoother gain matrices. Equation (20) and the definitions of $F_{i}$ and $L_{i}$ yield

$$
\begin{aligned}
& \mathrm{L}_{i}=\mathrm{H}_{i} \mathrm{~S}_{\mathrm{O}_{i}} \mathrm{H}_{i}^{\prime}+\mathrm{H}_{i} \mathrm{~S}_{1_{i}} \mathrm{G}_{i}^{\prime}+\mathrm{G}_{i} \mathrm{~S}_{1_{i}} \mathrm{H}_{i}^{\prime}+\mathrm{G}_{i} \mathrm{~S}_{2} \mathrm{G}_{i}^{\prime}+\Psi_{i}>0, \\
& \mathrm{M}_{i}=\mathrm{s}_{0_{i}} \mathrm{H}_{i}^{\prime}+\mathrm{s}_{1_{i}} \mathrm{G}_{i}^{\prime}, \\
& \mathrm{N}_{i}=\mathrm{s}_{1_{i}} \mathrm{H}_{i}^{\prime}+\mathrm{s}_{2} \mathrm{G}_{i}^{\prime} .
\end{aligned}
$$

We now expand (19) to arrive at a recursive system for the submatrices of $\mathrm{S}_{\mathbf{i}}$ : 


$$
\begin{aligned}
& S_{0}(\theta+1, \phi+1)=S_{0}(\theta, \phi)-M_{i}(\theta) L_{i}^{-1} M_{i}^{\prime}(\phi), \\
& s_{1}(\theta+1, \phi+1)=s_{1}(\theta, \phi)-M_{i}(\theta) L_{i}^{-1} N_{i}^{\prime}(\phi) \text {, } \\
& S_{2}(\theta+1, \phi+1)=S_{2}(\theta, \phi)-N_{i}(\theta) L_{i}^{-1} N_{i}^{\prime}(\phi)
\end{aligned}
$$

(again with indices $\theta, \phi$ taken over the appropriate range 0 to $k$, or 1 to $\mathrm{h})$. Note that $s_{0}(\theta+1, \phi+1)$ and $s_{0}(\theta, \phi)$ are both the estimate-error covariance matrices for $x_{i-\theta}, x_{i-\phi}$. But $s_{0_{i+1}}$ is conditioned on $z_{i}$, while $S_{0_{i}}$ is conditioned on $z_{i-1}$.

Equations (28) therefore update the covariance of the history estimates. Since the negative term is positive semidefinite, the covariances cannot increase as additional information is brought in.

The equations (28) are used with the expanded form of (19) to yield the error covariance matrices of the prediction $\hat{x}_{i+1 \mid i}$ and $\hat{u}_{i \mid i}$ with the other estimates:

$$
\begin{aligned}
& \mathrm{S}_{0}(0,0)=\sum_{i=0}^{k} \sum_{\phi=0}^{k} A_{i}(\theta) S_{0}(\theta+1, \phi+1) A_{i}^{\prime}(\phi)+\sum_{\theta=0}^{k} \sum_{\phi=1}^{h} A_{i}(\theta) S_{1}(\theta+1, \phi+1) B_{i}^{\prime}(\phi) \\
& +\sum_{\theta=1}^{h} \sum_{\phi=0}^{k} B_{i}(\theta) S_{1}^{\prime}(\theta+1, \phi+1) A_{i}^{\prime}(\phi)+\sum_{\theta=1}^{h} \sum_{\phi=1}^{h} B_{i}(\theta) S_{2}(\theta+1, \phi+1) B_{i}^{\prime}(\phi)+\Lambda_{i} \text {, } \\
& S_{0}(0, \phi)=\sum_{\theta=0}^{k} A_{i}(\theta) S_{0}(\theta+1, \phi)+\sum_{\theta=1}^{h} B_{i}(\theta) S_{i+1}^{\prime}(\theta+1, \phi), \phi=1, \ldots, k
\end{aligned}
$$$$
S_{1}(0,1)=\sum_{\theta=0}^{k} A_{i}(\theta) S_{1}(\theta+1,1)+\sum_{\theta=1}^{h} B_{i}(\theta) S_{2}(\theta+1,1)+C_{i+1}^{T} T_{i},
$$

$S_{1}(0, \phi)=\sum_{\theta=0}^{k} A_{i}(\theta) S_{1}(\theta+1, \phi)+\sum_{\theta=1}^{h} B_{i}(\theta) S_{2}(\theta+1, \phi), \phi=2, \ldots, h$

$S_{2}(1,1)=T_{i} ; \quad$ otherwise $S_{1}, S_{2}=0$ 
Recursion of $S$ is initialized by equating $S_{0}(\theta, \phi), S_{1}(\theta, \phi)$, $S_{2}(\theta, \phi)$ at time 0 to $\operatorname{Cov}\left(x_{-\theta}, x_{-\phi}\right), \operatorname{Cov}\left(x_{-\theta}, u_{-\phi}\right), \operatorname{Cov}\left(u_{-\theta}, u_{-\phi}\right)$. Since filter gain and covariance equations do not depend on realtime values, they may be computed in advance. Only the pasthistory estimates need be computed on line.

The filter-smoother derived above specializes to that of Mishra and Rajamani (1975) for the state-variable distributedlag case they consider.

\section{REMARKS AND EXTENSIONS}

We have obtained an optimal controller and estimator expressed in terms of the original problem. The resulting gain matrix expressions in (22), (23) and (27) to (29) seem more lengthy than those for the equivalent problem, but they require multiplications of order $(n k+m h+n)^{2}$ rather than $(n k+m h+n)^{3}$ at each step.

The time-lag structure of the controller and estimator is clear from (21) and (24) to (26). In contrast to the no-lag case, the controller does not use a once-only estimate of each variable; instead it exploits the fact that lagged variables remain in the dynamics for some time, and during this time the system can "learn" by mixing in new information. For this reason, if estimation lags are shorter than dynamics lags, estimation must still proceed back to the dynamics lag-limits. The controller acts on changing but constantly improving laggedvariable estimates. Note that in cases of informational delay the estimator is constructed to "predict" those lagged variables that have not yet entered direct observation. These "predictions" improve as time progresses.

The discrete-time matrix Riccati results above correspond almost term by term to those for the continuous-time case. Extra terms are present however due to the discrete time interval. It is therefore not possible to obtain the discrete results by discretization of the continuous results; it is possible, however, 
to go in the other direction. The discrete results can yield the continuous ones by appropriate passage to the limit (see Arthur (1977)).

Some extensions of the problem are worth noting briefly. For example the results are easily modified to the case of a time-lagged criterion. Also, varying lag-limits may be accommodated by replacing $k$ and $h$ by $k(i)$ and $h(i)$, provided $k(i)$ and $h(i)$ do not lengthen by more than one unit per unit time. otherwise the maximum lag duration can serve as $k$ or $h$.

The above results carry over to the infinite-horizon, timeinvariant regulator case as long as the properties strong controllability and strong observability are met. That is, we must be able to simultaneously control and consistently estimate not just the present state $x_{i}$ but the entire history, $x_{i}, \ldots, x_{i-k}$, $u_{i-1}, \ldots, u_{i-h}$. (Cf. for example Thowsen (1977), or Delfour and Mitter (1972).) These properties then guarantee (a) existence of optimal controls and optimal estimator given an infinite horizon, (b) asymptotic stability of the closed estimatorfeedback controller system, (c) convergence of the gain matrices to stationary values.

\section{CONCLUSIONS}

Discrete-time stochastic control results were presented for LQG problems with distributed lags in dynamics and observations. Optimal controls are linear in the estimates of past states and controls, and an optimal filter-smoother obtains and updates these estimates in linear fashion. Gain-matrix calculations are faster than in the usual high-dimensional methods, and the discrete-time results show close correspondence to those for the continuous-time case.

\section{ACKNOWLEDGEMENTS}

The author would like to thank S.E. Dreyfus and M. Ferguson for helpful comments. 


\section{REFERENCES}

Aoki, M. (1976), Optimal Control and System Theory in Dynamic Economic Analysis, American Elsevier - North Holland, New York, p. 26.

Arthur, W.B. (1977), The Control of Linear Processes with Distributed Lags using Dynamic Programming from First Principles, J. Optim. Theor. Appl., 23, forthcoming.

Chow, G. (1975), Analysis and Control of Dynamic Economic systems, Wiley-Interscience, New York.

Delfour, M.C., and S.K. Mitter (1972), Controllability, Observability and optimal Feedback Control of Affine Hereditary Differential systems, SIAM J. Contr., 10, 298-329.

Koivo, A.J. (1974), Optimal Control of Linear Stochastic Systems Described by Functional Differential Equations, J. Optim. Theor. Appl., 9, 161-175.

Kwong, R.H., and A.S. Willsky (1977), Optimal Filtering and Filter Stability of Linear Stochastic Delay Systems, I.E.E.E. Trans. Automat. Contr., AC-22, 196-202.

Meier, L., R.E. Larson, and A.J. Tether (1971), Dynamic Programming for the Stochastic Control of Discrete Systems, I.E.E.E. Trans. Automat. Contr., AC-16, 767-775.

Mishra, J., and V.S. Rajamani (1975), Least-Squares State Estimation in Time-Delayed Systems with Colored observation Noise: an Innovations Approach, I.E.E.E. Trans. Automat. Contr., AC-20, 140-142.

Thowsen, A. (1977), On Pointwise Degeneracy, Controllability and Minimum Time Control of Linear Dynamical Systems with Delays, Internat. J. Contr., 25, 329-345. 